Article

\title{
Bioactivity-Guided Fractionation of Physical Fatigue-Attenuating Components from Rubus parvifolius $\mathrm{L}$.
}

\author{
Jianhong Chen, Xianfeng Wang, Yongqing Cai, Ming Tang, Qing Dai, Xiaogang Hu, \\ Mingchun Huang, Fengjun Sun, Yao Liu and Peiyuan Xia *
}

Department of Pharmacy, Southwest Hospital, Third Military Medical University, Chongqing 400038, China; E-Mails: chenjh-110@263.net(J.C.); wxf3111@126.com (X.W.); cy0721@163.com (Y.C.); tangmin1007@163.com (M.T.); dq050@163.com (Q.D.); hxgcq1987@126.com (X.H.); xyjk11@sina.com (M.H.); fengj_sun@163.com (F.S.); lyly11982@hotmail.com (Y.L.)

* Author to whom correspondence should be addressed; E-Mail: py_xia2013@163.com; Tel.: +86-23-6875-4438; Fax: +86-23-6546-1719.

Received: 19 July 2013; in revised form: 25 August 2013 / Accepted: 6 September 2013 / Published: 23 September 2013

\begin{abstract}
Alleviation of fatigue has been emerging as a serious issue that requires urgent attention. Health professionals and sports physiologists have been looking for active natural products and synthetic compounds to overcome fatigue in humans. This study was designed to define the anti-fatigue property of Rubus parvifolius L. (RPL) by characterization of active constituents using a mouse forced swimming test model. Four RPL fractions with different polarities containing anti-fatigue activity were sequentially isolated from the $n$-butanol RPL extract, followed by elution of $50 \%$ ethanol-water fraction from D101 macroporous resin chromatography to obtain nigaichigoside $\mathrm{F}_{1}$, suavissimoside $\mathrm{R}_{1}$ and coreanoside $\mathrm{F}_{1}$. Active constituents of the $50 \%$ ethanol-water eluate of RPL were total saponins. The fractions were examined based on the effect on weight-loaded swimming capacity of mice. Serum levels of urea nitrogen (SUN), triglyceride fatty acids (TG), lactate dehydrogenase (LDH), lactic acid (LA), ammonia and hepatic glycogen (HG) were also examined for potential mechanisms underlying the anti-fatigue effect of RPL extracts. During the experiment, two inflammatory markers, interleukin-6 (IL-6) and tumor necrosis factor (TNF- $\alpha$ ) in serum, were measured. We found that total saponins from RPL possess potent capabilities to alleviate mouse fatigue induced by forced swimming and that nigaichigoside $F_{1}$ was responsible for the pharmacological effect. The underlying mechanisms include delays of SUN and LA accumulation, a decrease in TG level by increasing fat consumption, increases in $\mathrm{HG}$ and $\mathrm{LDH}$ so that lactic acid accumulation and
\end{abstract}


ammonia in the muscle were reduced, and suppression of increased immune activation and inflammatory cytokine production. Our findings will be helpful for functional identification of novel anti-fatigue components from natural medicinal herbs.

Keywords: Rubus parvifolius L.; anti-fatigue; macroporous resin; weight-loaded swimming

\section{Introduction}

Rubus parvifolius L. (RPL), belonging to the genus Rubus, is a deciduous thorny shrub, native to the eastern and southern regions of Asia. It has been used as an anti-inflammatory and anti-infectious herbal medicine for treatment of a variety of diseases including rheum, dysentery, enteritis, angina, hepatitis, rheumatism, dermatitis and eczema [1]. Previous studies demonstrated that RPL extracts exhibited antioxidant capacity [2-4], neuroprotective effects [5,6] and anti-tumor properties [7].

Fatigue is defined as difficulty in initiating or sustaining voluntary activities, resulting from severe stress and hard physical or mental work [8,9]. Therefore, alleviation of fatigue has become a serious issue that requires urgent attention. In the past few decades, health professionals and sports physiologists have been looking for active natural products and synthetic chemicals to postpone, and accelerate the elimination of, fatigue in humans [10]. However, effective drugs with few side effects are limited. The aim of the present study was to examine the anti-fatigue properties of the aqueous ethanol extract of RPL, its fractions and isolated compounds to provide a scientific basis for the industrial production and clinical use of RPL in a mouse forced swimming test model. We further examined the levels of mouse serum urea nitrogen (SUN), triglyceride fatty acids (TG), lactate dehydrogenase (LDH), lactic acid (LA), ammonia and hepatic glycogen (HG) to elucidate the mechanisms underlying the anti-fatigue effect of RPL. Two inflammatory markers interleukin-6 (IL-6) and tumor necrosis factor $(\mathrm{TNF}-\alpha)$ in serum were also examined.

\section{Results and Discussion}

\subsection{Chemical Analysis}

From the $50 \%$ ethanol fraction eluated from macroporous resin, three saponins (Figure 1A) were obtained and identified as nigaichigoside $F_{1}$ (compound $I$, Figure 1B,C), suavissimoside $R_{1}$ (compound J, Figure 1C) and coreanoside $F_{1}$ (compound K, Figure 1D), both of which have been previously reported and isolated from the leaves of Rubus species [11-13]. The structures were elucidated unambiguously by comparing experimental data with literature data. 
Figure 1. HPLC fractions from Rubus parvifolius L. (A) HPLC fingerprint of 50\% ethanol fraction. (B) HPLC of 50\% ethanol fraction isolate (nigaichigoside $F_{1}$ ). (C) Chemical structure of nigaichigoside $F_{1}$ (I) suavissimoside $R_{1}$ (J). (D) Chemical structure of coreanoside F1 (K).

(A)

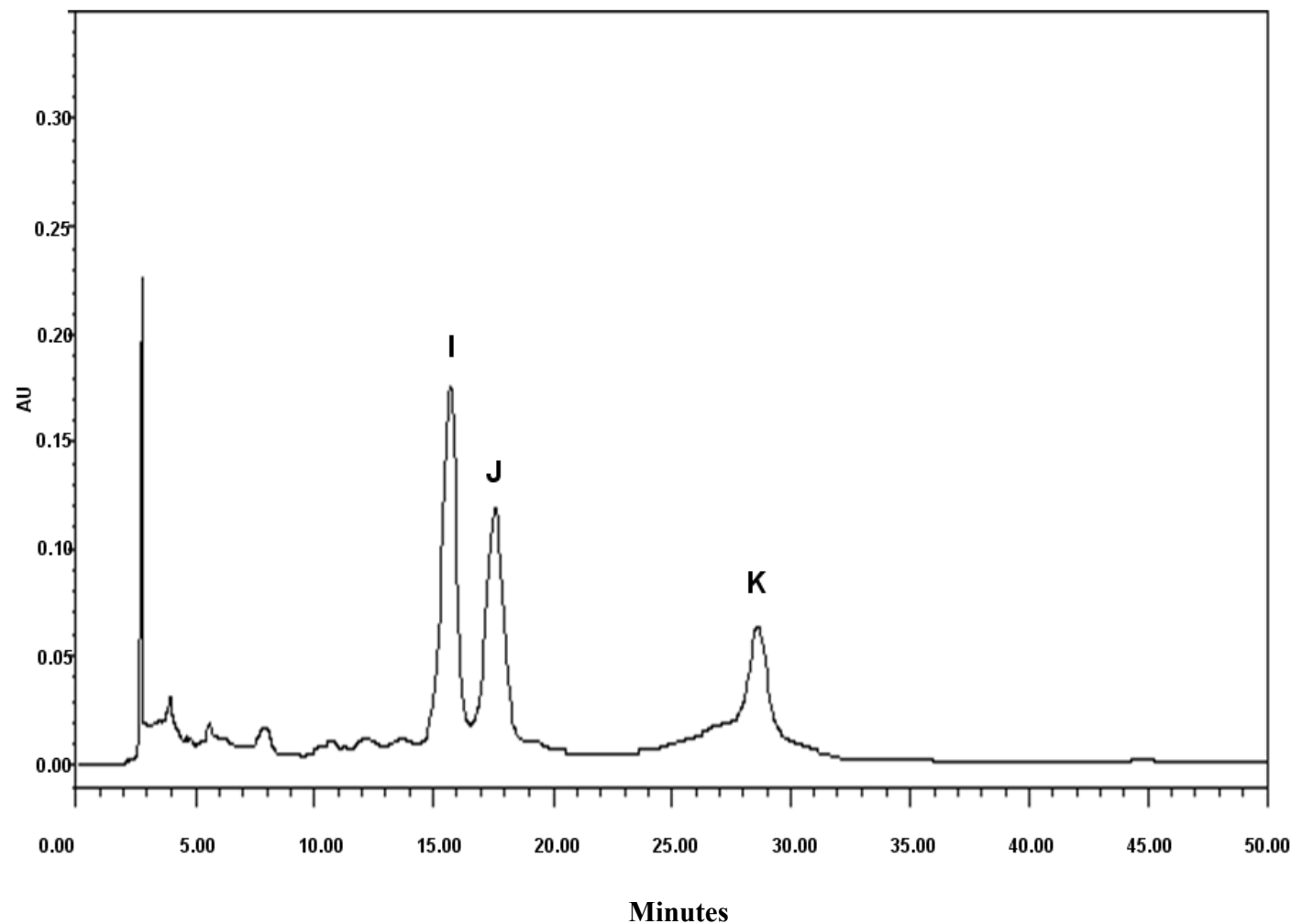

(B)

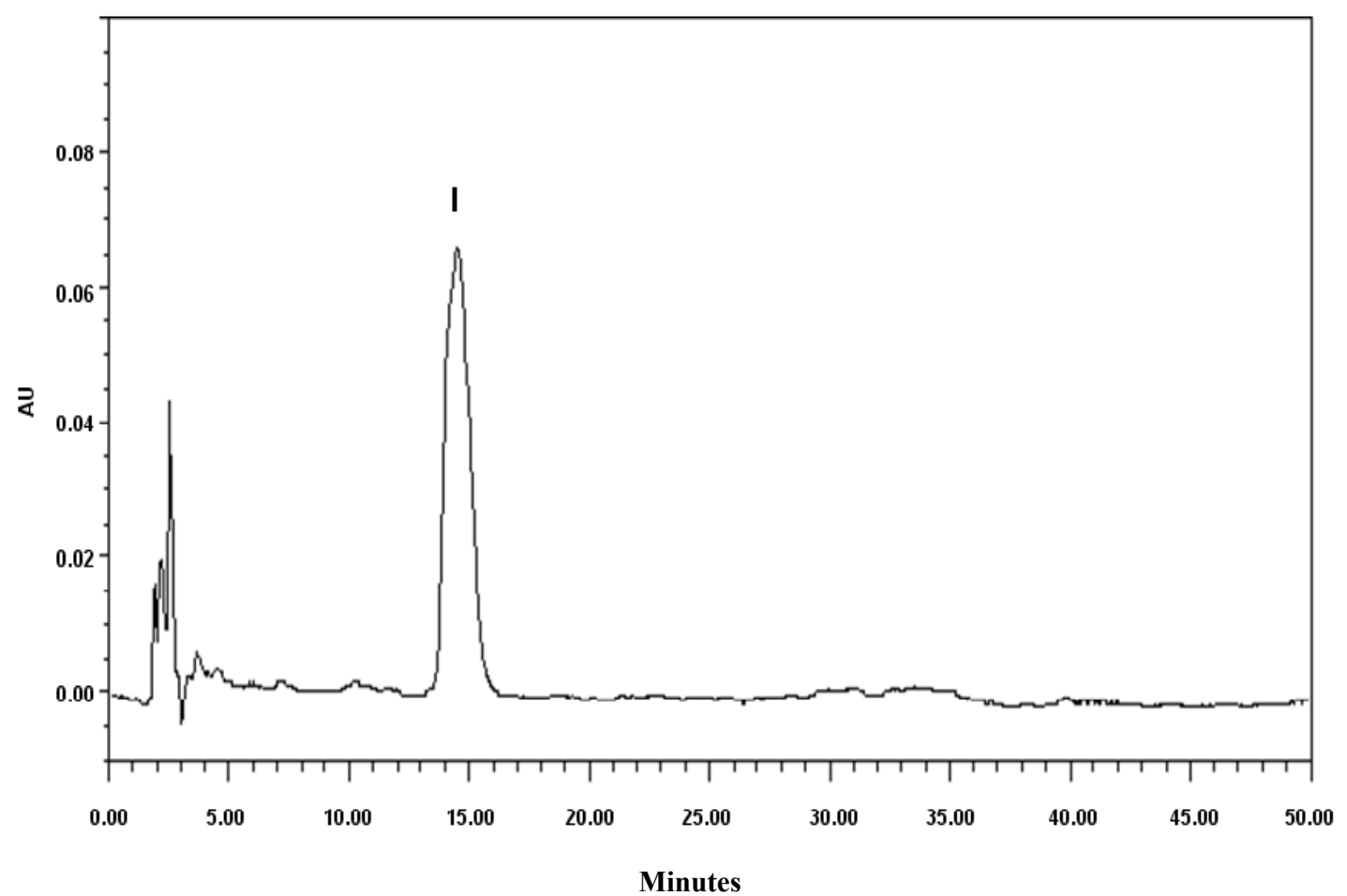


Figure 1. Cont.

(C)

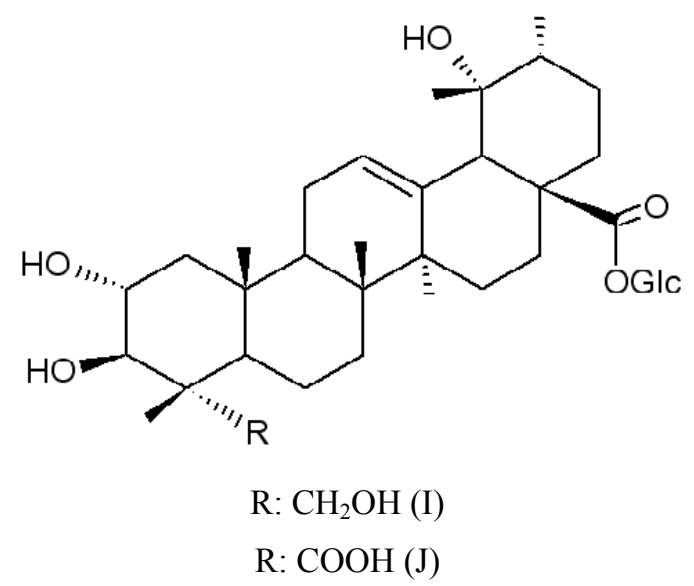

(D)

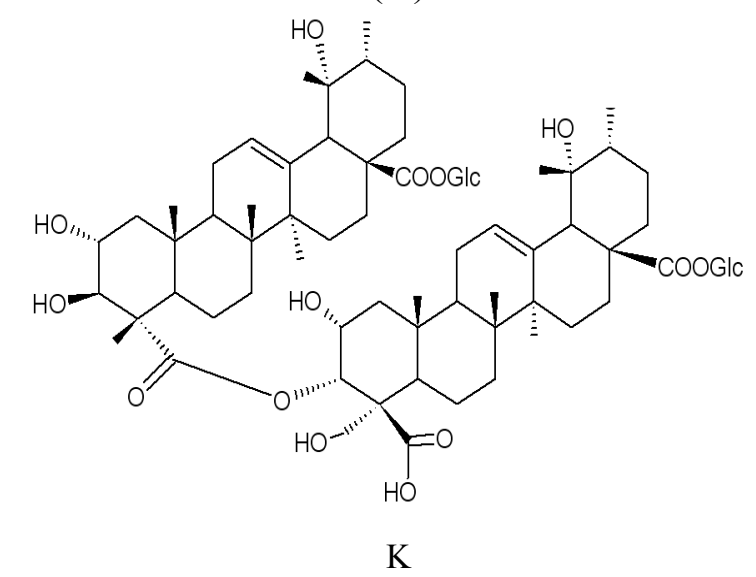

\subsection{The Effects of RPL on Mouse Body Weight Change}

We compared the effects of red ginseng and different solvent fractions of RPL on mouse body weight at initial and terminal stages of the swimming test. As shown in Table 1, at the end of administration, there was an increase in the body weight of the mice in all groups.

No significant difference in the body weight was found in treatment groups as compared with control group during initial and terminal stages of the animal experiments I and II. The effects of $n$-butanol fractions and nigaichigoside $\mathrm{F}_{1}$ on body weight were also compared.

Table 1. The effects of the fractions of RPL and red ginseng on mice body weights.

\begin{tabular}{cccc}
\hline \multirow{2}{*}{ Groups } & \multicolumn{3}{c}{ Body weights(g) } \\
\cline { 2 - 4 } & Initial & Final & Increased \\
\hline Control & $23.8 \pm 0.5$ & $40.2 \pm 0.8$ & $16.4 \pm 0.8$ \\
Red Ginseng & $24.0 \pm 0.7$ & $39.6 \pm 0.8$ & $15.6 \pm 0.9$ \\
A1 & $24.4 \pm 1.0$ & $40.5 \pm 0.9$ & $16.1 \pm 1.2$ \\
A2 & $23.7 \pm 0.9$ & $39.6 \pm 1.3$ & $15.9 \pm 1.2$ \\
B1 & $24.2 \pm 0.9$ & $39.8 \pm 1.3$ & $15.6 \pm 1.0$ \\
B2 & $23.6 \pm 0.9$ & $39.3 \pm 1.1$ & $15.7 \pm 0.8$ \\
C1 & $24.2 \pm 1.0$ & $40.6 \pm 1.2$ & $16.3 \pm 0.9$ \\
C2 & $23.3 \pm 0.9$ & $39.5 \pm 1.2$ & $16.1 \pm 1.4$ \\
D1 & $24.5 \pm 1.0$ & $41.1 \pm 1.3$ & $16.6 \pm 1.1$ \\
D2 & $24.1 \pm 0.8$ & $39.9 \pm 1.2$ & $15.8 \pm 1.2$ \\
\hline
\end{tabular}

A1: low dose of petroleum ether fraction; A2: high dose of petroleum ether fraction; B1: low dose of ethyl acetate fraction; B2: high dose of ethyl acetate fraction; C1: low dose of $n$-butanol fraction; C2: high dose of $n$-butanol fraction; D1: low dose of water reside; D2: high dose of water reside; Low dose denotes $20 \mathrm{mg} / \mathrm{kg}$; High dose denotes $40 \mathrm{mg} / \mathrm{kg}$ for each mouse. 
As shown in Table 2, there were no significant differences in the body weight of the treatment groups as compared with the control group during initial and terminal stages in the mouse experiments I and II, although there were increases in the body weight of the mice in all groups. Thus, the compounds and derivates do not change the natural body weight gain of mice.

Table 2. The effects of the $n$-butanol fraction and nigaichigoside $\mathrm{F}_{1}$ on mice body weights.

\begin{tabular}{cccc}
\hline \multirow{2}{*}{ Groups } & \multicolumn{3}{c}{ Body weights(g) } \\
\cline { 2 - 4 } & Initial & Final & Increased \\
\hline Normal & $23.2 \pm 0.6$ & $31.2 \pm 0.8$ & $8.1 \pm 0.7$ \\
Control & $23.3 \pm 0.5$ & $31.3 \pm 0.6$ & $8.0 \pm 0.6$ \\
RG1 & $23.8 \pm 0.8$ & $31.2 \pm 0.7$ & $7.4 \pm 0.8$ \\
RG2 & $24.0 \pm 0.7$ & $31.9 \pm 0.6$ & $7.9 \pm 0.8$ \\
E1 & $23.7 \pm 0.9$ & $31.6 \pm 0.9$ & $7.9 \pm 0.7$ \\
E2 & $23.9 \pm 0.9$ & $31.6 \pm 0.7$ & $7.7 \pm 1.0$ \\
F1 & $22.8 \pm 0.8$ & $31.2 \pm 0.6$ & $8.4 \pm 1.1$ \\
F2 & $23.5 \pm 0.7$ & $30.9 \pm 0.6$ & $7.4 \pm 0.7$ \\
G1 & $23.7 \pm 0.9$ & $31.0 \pm 1.0$ & $7.3 \pm 0.8$ \\
G2 & $23.2 \pm 0.8$ & $30.9 \pm 0.9$ & $7.7 \pm 0.9$ \\
H1 & $23.1 \pm 0.8$ & $30.6 \pm 0.8$ & $7.6 \pm 0.7$ \\
H2 & $22.9 \pm 0.8$ & $30.8 \pm 0.9$ & $7.9 \pm 1.1$ \\
I1 & $24.0 \pm 1.0$ & $32.1 \pm 1.0$ & $8.1 \pm 0.9$ \\
I2 & $23.7 \pm 0.8$ & $31.1 \pm 0.9$ & $7.5 \pm 0.9$ \\
\hline
\end{tabular}

Normal: non-swimming control group; Control: swimming control group; RG1: low dose of red ginseng; RG2: high dose of red ginseng; E1: low dose of water eluate; E2: high dose of water eluate; F1: low dose of $20 \%$ ethanol eluate; F2: high dose of $20 \%$ ethanol eluate; G1: low dose of $50 \%$ ethanol eluate; G2: high dose of $50 \%$ ethanol eluate; H1: low dose of $100 \%$ ethanol eluate; H2: high dose of $100 \%$ ethanol eluate; $\mathrm{I} 1$ and $\mathrm{I} 2$ mean that animals were treated with nigaichigoside $\mathrm{F}_{1} 2 \mathrm{mg} / \mathrm{kg}$ and $4 \mathrm{mg} / \mathrm{kg}$.

\subsection{The Effects of RPL on Mice in Swimming-to-Exhaustion Test}

We next examined the anti-fatigue effects of RPL and fractions on mice in a swimming-to-exhaustion test. In experiment I, the swimming time to exhaustion of mice receiving high-dose of the $n$-butanol fraction and the positive (red ginseng) group was significantly longer than that of the control group (Figure 2, $p<0.01$ ). In experiment II, the swimming time of mice treated with high-dose of $20 \%$ ethanol, $50 \%$ ethanol fractions and nigaichigoside $\mathrm{F}_{1}$ was significantly improved (Figure 3, $p<0.01$ ). Thus, RPL and its fractions are effective in increasing the swimming time to exhaustion, indicating the anti-fatigue activity of RPL compounds. 
Figure 2. The effects of isolated fractions from Rubus parvifolius L. and red ginseng on swimming time to exhaustion of weight-loaded mice. A1: low dose of petroleum ether fraction, A2: high dose of petroleum ether fraction, B1: low dose of ethyl acetate fraction, $\mathrm{B} 2$ : high dose of ethyl acetate fraction, $\mathrm{C} 1$ : low dose of $n$-butanol fraction, C2: high dose of n-butanol fraction, D1: low dose of water reside, and D2: high dose of water reside. Low dose denotes $20 \mathrm{mg} / \mathrm{kg}$ per mouse, and high dose denotes $40 \mathrm{mg} / \mathrm{kg}$ per mouse.

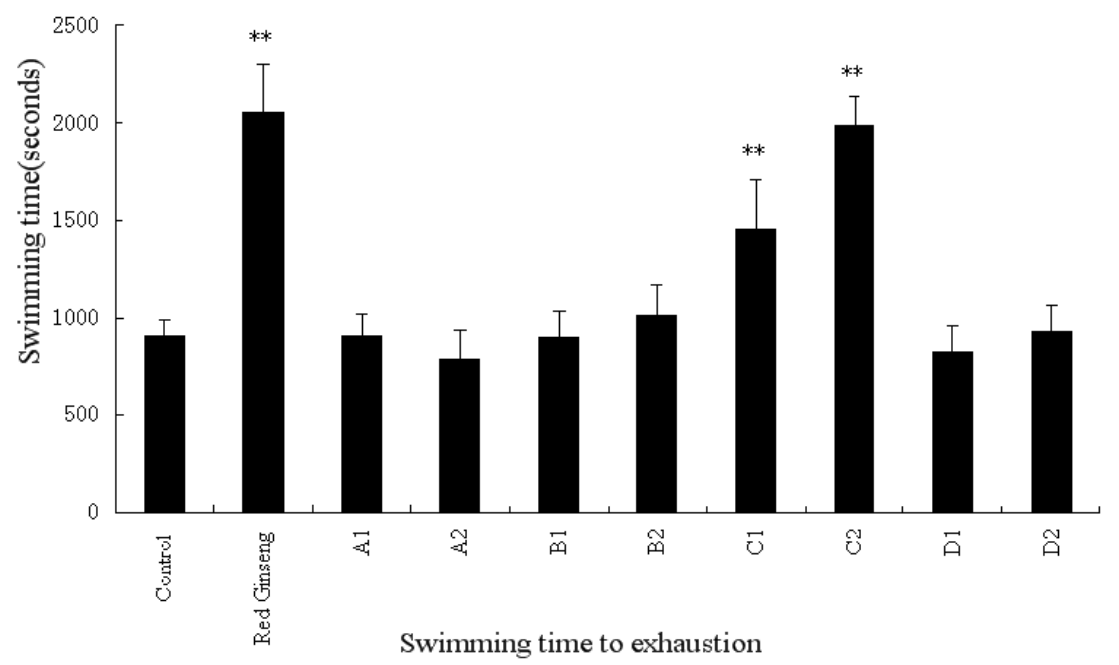

** indicates statistically significant difference as compared to the control group $(p<0.01)$.

Figure 3. The effects of n-butanol fractions on the swimming time to exhaustion of weight-loaded mice. RG1: low dose of red ginseng, RG2: high dose of red ginseng, E1: low dose of water eluate, E2: high dose of water eluate, F1: low dose of $20 \%$ ethanol eluate, F2: high dose of $20 \%$ ethanol eluate, G1: low dose of $50 \%$ ethanol eluate, G2: high dose of $50 \%$ ethanol eluate, $\mathrm{H} 1$ : low dose of $100 \%$ ethanol eluate, $\mathrm{H} 2$ : high dose of $100 \%$ ethanol eluate, I1: low dose of $100 \%$ ethanol eluate, and I2: high dose of $100 \%$ ethanol eluate. The fractions were collected from D101 macroporous resin. Low dose denotes $10 \mathrm{mg} / \mathrm{kg}$ per mouse, and high dose denotes $20 \mathrm{mg} / \mathrm{kg}$ per mouse. I1 and $\mathrm{I} 2 \mathrm{represent}$ animals treated with nigaichigoside $\mathrm{F} 1$ at $2 \mathrm{mg} / \mathrm{kg}$ and $4 \mathrm{mg} / \mathrm{kg}$, respectively.

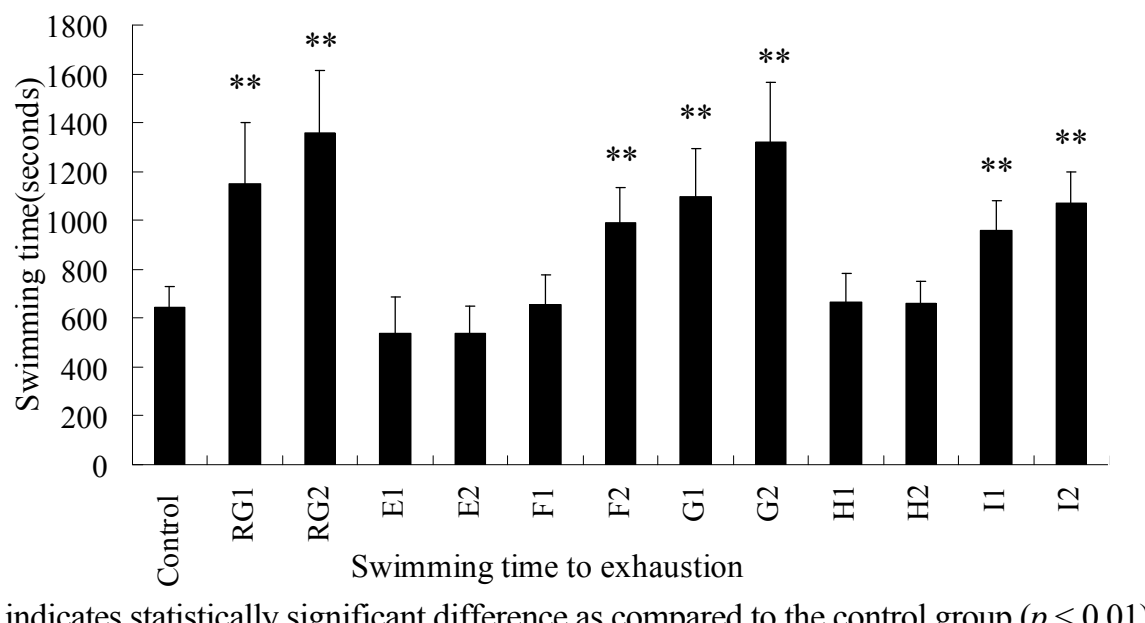

** indicates statistically significant difference as compared to the control group $(p<0.01)$. 


\subsection{The Effects of RPL on SUN, TG, LDH, Ammonia, LA and $H G$}

The concentrations of serum SUN, TG, LDH, ammonia and LA and hepatic glycogen (HG) serve as indicators of accumulated fatigue. As shown in Tables 3 and 4, the SUN levels were significantly decreased in the red ginseng group $(p<0.01)$, the high dose ethyl acetate fraction group $(p<0.05)$ in the mouse experiment I, the low dose $n$-butanol fraction group $(p<0.01)$, the high dose $n$-butanol fraction group $(p<0.01)$, the high dose water residue group $(p<0.05)$, the low dose $50 \%$ ethanol eluate group ( $p<0.05$ ), the high dose 50\% ethanol eluate group $(p<0.01)$, nigaichigoside $\mathrm{F}_{1} 4 \mathrm{mg} / \mathrm{kg}$ group and nigaichigoside $\mathrm{F}_{1} 2 \mathrm{mg} / \mathrm{kg}$ group $(p<0.05)$ in experiment II. The concentration of serum triglyceride (TG) in red ginseng, high-dose ethyl acetate, and low dose $n$-butanol fraction groups were lower than that in the control group $(p<0.05)$, with the high-dose $n$-butanol group being more highly significant $(p<0.01)$. In experiment II, the TG levels were also lower in red ginseng, the high-dose $20 \%, 50 \%$ ethanol fractions and nigaichigoside $\mathrm{F}_{1}$ groups as compared to control group $(p<0.05$ or $p<0.01)$. LDH change was not significantly different in all groups in the experiment I. However, in the experiment II, among the groups of the high-dose $20 \%$ ethanol fraction, and both doses of $50 \%$ ethanol fractions and nigaichigoside $\mathrm{F}_{1}$, the LDH level was significantly higher in comparison with the untreated group $(p<0.05)$. The serum ammonia levels in the high dose n-butanol fraction group were significant lower than those in the control group $(p<0.05)$ in experiment I.

Table 3. The effects of the fractions of RPL and red ginseng on SUN, TG, LDH and ammonia.

\begin{tabular}{ccccc}
\hline Groups & SUN (mmol/L) & TG $(\mathbf{m m o l} / \mathbf{L})$ & LDH $(\mathbf{I U} / \mathbf{L})$ & Ammonia (umol/L) \\
\hline Control & $10.33 \pm 0.63$ & $2.880 \pm 0.1052$ & $585.1 \pm 21.12$ & $93.1 \pm 7.29$ \\
Red Ginseng & $9.29 \pm 0.71^{* *}$ & $2.765 \pm 0.1246^{*}$ & $590.7 \pm 17.22$ & $91.9 \pm 6.71$ \\
A1 & $9.95 \pm 0.77$ & $2.875 \pm 0.0664$ & $575.2 \pm 23.64$ & $94.9 \pm 8.48$ \\
A2 & $9.74 \pm 0.76$ & $2.858 \pm 0.1055$ & $580.8 \pm 21.80$ & $98.3 \pm 8.51$ \\
B1 & $9.81 \pm 0.78$ & $2.931 \pm 0.1064$ & $594.6 \pm 28.41$ & $87.2 \pm 7.21$ \\
B2 & $9.04 \pm 0.66^{*}$ & $2.784 \pm 0.0763 *$ & $587.7 \pm 28.23$ & $90.5 \pm 6.38$ \\
C1 & $9.41 \pm 0.76^{* *}$ & $2.769 \pm 0.0568 *$ & $597.2 \pm 21.50$ & $88.1 \pm 6.67$ \\
C2 & $8.84 \pm 0.70^{* *}$ & $2.745 \pm 0.0573 * *$ & $603.3 \pm 27.45$ & $85.6 \pm 8.14 *$ \\
D1 & $9.72 \pm 0.68$ & $2.967 \pm 0.0934$ & $567.6 \pm 23.28$ & $94.7 \pm 9.03$ \\
D2 & $9.55 \pm 0.69 *$ & $2.909 \pm 0.1184$ & $578.4 \pm 24.91$ & $95.2 \pm 6.56$ \\
\hline
\end{tabular}

A1: low dose of petroleum ether fraction; A2: high dose of petroleum ether fraction; B1: low dose of ethyl acetate fraction; B2: high dose of ethyl acetate fraction; $\mathrm{C} 1$ : low dose of $n$-butanol fraction; C2: high dose of n-butanol fraction; D1: low dose of water reside; D2: high dose of water reside; Low dose denotes $20 \mathrm{mg} / \mathrm{kg}$; high dose denotes $40 \mathrm{mg} / \mathrm{kg}$ for each mouse; ${ }^{*} p<0.05$ and ${ }^{* *} p<0.01 v s$. control group.

In experiment II, among mice receiving high-dose $20 \%$ ethanol fraction, and both doses of the $50 \%$ ethanol fractions and nigaichigoside $\mathrm{F}_{1}$, their LDH levels were significantly higher in comparison with the untreated group $(p<0.05, p<0.01)$. LDH was increased by $n$-butanol and nigaichigoside $\mathrm{F}_{1}$ fractions. $\mathrm{LDH}$ is known to catalyze lactic acid into pyruvate, thereby decreasing the accumulation of lactic acid in muscle to attenuate fatigue $[10,14]$, although a previous report cited that liposoluble fraction from Acanthopanax senticosus decreased LDH [15]. The serum ammonia levels in mice treated with high-dose $20 \%$ ethanol fraction, and both doses of the $50 \%$ ethanol fractions and 
nigaichigoside $\mathrm{F}_{1}$ showed a significant decrease in experiment II, while in experiment I only high dose $n$-butanol fraction treated group showed a decrease in the serum ammonia levels. In experiment II, we also determined the levels of serum LA and HG, As shown in Table 4, the LA level was significantly decreased in the groups that were treated with high dose of red ginseng, 50\% ethanol eluate and nigaichigoside $\mathrm{F}_{1}(p<0.05$ or $p<0.01)$, while the concentration of HG was higher $(p<0.05$ or $p<0.01)$. These results confirm that RPL and its fractions possess anti-fatigue properties.

Table 4. The effects of the $n$-butanol and nigaichigoside $\mathrm{F}_{1}$ fractions on SUN, TG, LDH, ammonia, LA and TG.

\begin{tabular}{ccccccc}
\hline Groups & $\begin{array}{c}\text { BUN } \\
(\mathbf{m m o l} / \mathbf{L})\end{array}$ & TG $(\mathbf{m m o l} / \mathbf{L})$ & $\mathbf{L D H}(\mathbf{I U} / \mathbf{L})$ & $\begin{array}{c}\text { Ammonia } \\
(\mathbf{u m o l} / \mathbf{L})\end{array}$ & $\mathbf{L A}(\mathbf{m g} / \mathbf{d L})$ & $\mathbf{H G}(\mathbf{m g} / \mathbf{g})$ \\
\hline Normal & $6.48 \pm 0.45$ & $1.959 \pm 0.069$ & $383.9 \pm 23.27$ & $52.2 \pm 5.09$ & $44.1 \pm 4.6$ & $47.90 \pm 5.83$ \\
Control & $9.66 \pm 0.68$ & $2.162 \pm 0.083$ & $894.5 \pm 35.59$ & $134.1 \pm 7.26$ & $65.9 \pm 6.1$ & $19.44 \pm 1.96$ \\
RG1 & $9.06 \pm 0.86$ & $2.077 \pm 0.090 *$ & $919.9 \pm 60.71$ & $128.9 \pm 10.21$ & $61.3 \pm 5.6$ & $39.26 \pm 3.82 * *$ \\
RG2 & $8.91 \pm 0.71 *$ & $2.034 \pm 0.077 * *$ & $922.4 \pm 49.74$ & $127.4 \pm 9.62$ & $59.3 \pm 6.4 *$ & $43.17 \pm 5.38 * *$ \\
E1 & $9.38 \pm 0.70$ & $2.148 \pm 0.077$ & $927.5 \pm 50.40$ & $129.7 \pm 9.32$ & $62.2 \pm 6.9$ & $19.76 \pm 1.93$ \\
E2 & $9.66 \pm 0.84$ & $2.134 \pm 0.053$ & $921.1 \pm 43.57$ & $127.7 \pm 12.56$ & $61.9 \pm 8.7$ & $20.20 \pm 1.72$ \\
F1 & $9.41 \pm 0.63$ & $2.083 \pm 0.123$ & $908.3 \pm 38.90$ & $126.2 \pm 10.50$ & $64.6 \pm 8.8$ & $20.60 \pm 2.47$ \\
F2 & $9.21 \pm 0.75$ & $2.052 \pm 0.092 *$ & $932.5 \pm 42.57 *$ & $121.1 \pm 15.02 *$ & $63.8 \pm 8.3$ & $19.30 \pm 2.06$ \\
G1 & $8.90 \pm 0.71 *$ & $2.050 \pm 0.096 *$ & $938.2 \pm 47.62 *$ & $115.7 \pm 12.94 * *$ & $57.8 \pm 7.1 *$ & $40.59 \pm 4.19 * *$ \\
G2 & $8.58 \pm 0.69 * *$ & $1.992 \pm 0.074 * *$ & $970.5 \pm 81.40 *$ & $104.1 \pm 11.62 * *$ & $54.5 \pm 6.3 * *$ & $43.04 \pm 4.59 * *$ \\
H1 & $9.30 \pm 0.82$ & $2.165 \pm 0.104$ & $903.2 \pm 33.03$ & $125.7 \pm 14.64$ & $63.4 \pm 8.1$ & $21.58 \pm 3.34$ \\
H2 & $9.14 \pm 0.98$ & $2.178 \pm 0.125$ & $916.2 \pm 30.97$ & $135.1 \pm 11.28$ & $62.2 \pm 7.1$ & $21.89 \pm 3.60$ \\
I1 & $9.09 \pm 0.83$ & $2.170 \pm 0.103$ & $937.0 \pm 44.94 *$ & $113.3 \pm 11.54 * *$ & $60.9 \pm 4.3 *$ & $38.67 \pm 3.24 * *$ \\
I2 & $8.79 \pm 0.76 *$ & $2.067 \pm 0.107 *$ & $951.9 \pm 58.00 *$ & $109.0 \pm 15.19 * *$ & $58.7 \pm 5.7 *$ & $41.45 \pm 3.81 * *$ \\
\hline
\end{tabular}

Normal: non-swimming control group; Control: swimming control group; RG1: low dose of red ginseng; RG2: high dose of red ginseng; E1: low dose of water eluate; E2: high dose of water eluate; F1: low dose of $20 \%$ ethanol eluate; F2: high dose of $20 \%$ ethanol eluate; G1: low dose of $50 \%$ ethanol eluate; G2: high dose of $50 \%$ ethanol eluate; $\mathrm{H} 1$ : low dose of $100 \%$ ethanol eluate; $\mathrm{H} 2$ : high dose of $100 \%$ ethanol eluate; I1 and I2 mean that animals were treated with nigaichigoside $\mathrm{F}_{1} 2 \mathrm{mg} / \mathrm{kg}$ and $4 \mathrm{mg} / \mathrm{kg} ; * p<0.05$ and $* * p<0.01$ vs. control group.

\subsection{The Effects of RPL on IL-6 and TNF- $\alpha$}

Fatigue is associated with increased immune activation and the production of inflammatory cytokines $[16,17]$. Therefore, we analyzed two inflammatory cytokines in experiment II. As shown in Table 5, the serum level of IL-6 was significantly decreased in the groups that were treated with high dose of red ginseng, $50 \%$ ethanol eluate and nigaichigoside $\mathrm{F}_{1}(p<0.05)$. The concentration of TNF- $\alpha$ was also lower in the groups receiving red ginseng, 50\% ethanol fractions and nigaichigoside $\mathrm{F}_{1}$ groups as compared to control group ( $p<0.05$ or $p<0.01$ ). These results confirm that RPL and its fractions possess anti-fatigue properties through suppression of pro-inflammatory responses. 
Table 5. The effects of the $n$-butanol and nigaichigoside $F_{1}$ fractions on IL- 6 and TNF- $\alpha$.

\begin{tabular}{ccc}
\hline Groups & IL-6 $(\mathbf{p g} / \mathbf{m L})$ & TNF- $\boldsymbol{\alpha}(\mathbf{p g} / \mathbf{m L})$ \\
\hline Normal & $224.19 \pm 19.51 * *$ & $870.08 \pm 53.65 * *$ \\
Control & $348.04 \pm 25.39$ & $978.43 \pm 52.14$ \\
RG1 & $328.97 \pm 17.46$ & $926.13 \pm 34.58 *$ \\
RG2 & $320.11 \pm 19.94 *$ & $905.50 \pm 44.7 * *$ \\
E1 & $334.61 \pm 26.80$ & $941.72 \pm 47.44$ \\
E2 & $340.64 \pm 18.67$ & $945.96 \pm 73.91$ \\
F1 & $328.69 \pm 30.19$ & $956.67 \pm 53.94$ \\
F2 & $339.75 \pm 26.34$ & $945.39 \pm 75.32$ \\
G1 & $317.34 \pm 29.03 *$ & $912.73 \pm 35.94 * *$ \\
G2 & $310.84 \pm 32.74 *$ & $907.71 \pm 39.16 * *$ \\
H1 & $337.22 \pm 34.54$ & $937.43 \pm 45.74$ \\
H2 & $343.11 \pm 33.55$ & $947.29 \pm 49.97$ \\
I1 & $325.76 \pm 19.94 *$ & $931.77 \pm 38.32 *$ \\
I2 & $324.21 \pm 17.17 *$ & $923.91 \pm 44.69 *$ \\
\hline
\end{tabular}

Normal: non-swimming control group; Control: swimming control group; RG1: low dose of red ginseng; RG2: high dose of red ginseng; E1: low dose of water eluate; E2: high dose of water eluate; F1: low dose of $20 \%$ ethanol eluate; F2: high dose of $20 \%$ ethanol eluate; G1: low dose of 50\% ethanol eluate; G2: high dose of $50 \%$ ethanol eluate; $\mathrm{H} 1$ : low dose of $100 \%$ ethanol eluate; $\mathrm{H} 2$ : high dose of $100 \%$ ethanol eluate; I1 and I2 mean that animals were treated with nigaichigoside $\mathrm{F}_{1} 2 \mathrm{mg} / \mathrm{kg}$ and $4 \mathrm{mg} / \mathrm{kg} ;{ }^{*} p<0.05$ and ${ }^{* *} p<0.01 v s$. control group.

\section{Experimental}

\subsection{Chemicals, Reagents and Instruments}

The following reagents were used in this study: EtOH (AR), petroleum ether (AR), ethyl acetate (AR), and $n$-butanol (AR) (Chongqing Chuandong Chemical Co. Ltd., Chongqing, China). D101 macroporous resin was purchased from Chemical Plant of Nankai University (Tianjin, China), and prepared according to the manufaturers instructions. Briefly, the resin was immersed in $95 \%$ ethanol for $24 \mathrm{~h}$, washed by distilled water, and then washed by $5 \% \mathrm{HCl}, 5 \% \mathrm{NaOH}$ and distilled water thoroughly [18] before use. HPLC analysis of calibration solutions and those of extracts and fractions of Rubus parvifolius L. (RPL) was performed on a Waters modular chromatograph (Waters Corporation, Milford, MA, USA). This system was controlled by Millennium 32 software. The system consisted of a Waters-2690 binary pump and a photo diode array detector (PDA) model 996. HPLC separations were performed on a YMC-Pack ODS-AM column $(4.6 \times 250 \mathrm{~mm}, 5 \mu \mathrm{m})$ (YMC Co. Ltd., Tokyo, Japan) and a YMC-Pack ODS-AM column $(10 \times 250 \mathrm{~mm}, 10 \mu \mathrm{m})$. The samples were eluted with $\mathrm{MeOH}$ and a solution containing ultrapure $\mathrm{H}_{2} \mathrm{O}$ (Milli-Q, Millipore, Billerica, MA, USA) and formic acid (FA, 1\%). ${ }^{1} \mathrm{H}$ and ${ }^{13} \mathrm{C}$-NMR spectra were obtained on a Bruker Avance-500 spectrometer (Bruker, Karlsruhe, BW, Germany). 


\subsection{Plant Materials}

The whole plants of RPL were obtained from Chongqing Huiyuan Pharma. Co., Ltd. (Chongqing, China) Red ginseng pieces were purchased from Chongqing Tongjunge Pharmacy Chainstore (Chongqing, China). All plants were authenticated at the Chongqing Institute of Chinese Materia Medica.

\subsection{Extraction, Isolation of RPL Components}

Powdered whole plant of RPL (20 kg) was extracted and fractionated as illustrated the flow chart in Figure 4. Powdered whole plant of RPL ( $20 \mathrm{~kg}$ ) was soaked in $70 \%$ ethanol aqueous solution $(40 \mathrm{~L})$ for $24 \mathrm{~h}$, and then extracted using 10 volumes of $70 \%$ ethanol aqueous solution by percolation, the percolation rate was $300 \mathrm{~mL} /(\mathrm{kg} \cdot \mathrm{min})$ extraction. The solvent was evaporated under vacuum to yield the crude extract $(1,752 \mathrm{~g})$. This extract was then suspended in water and partitioned successively with petroleum ether $(3 \times 5 \mathrm{~L})$, ethyl acetate $(3 \times 5 \mathrm{~L})$ and water-saturated $n$-butanol $(4 \times 5 \mathrm{~L})$. Each fraction was evaporated to dryness under reduced pressure to afford the petroleum ether (A, $74 \mathrm{~g})$, ethyl acetate (B, $451 \mathrm{~g}), n$-butanol $(\mathrm{C}, 446 \mathrm{~g})$ and aqueous $(\mathrm{D}, 603 \mathrm{~g})$ residues. The extracts were concentrated under vacuum at $40{ }^{\circ} \mathrm{C}$, lyophilized to obtain powders, and then used as test samples.

Figure 4. Schematic description of the procedures for extraction and fractionation of the whole plant of Rubus parvifolius L.

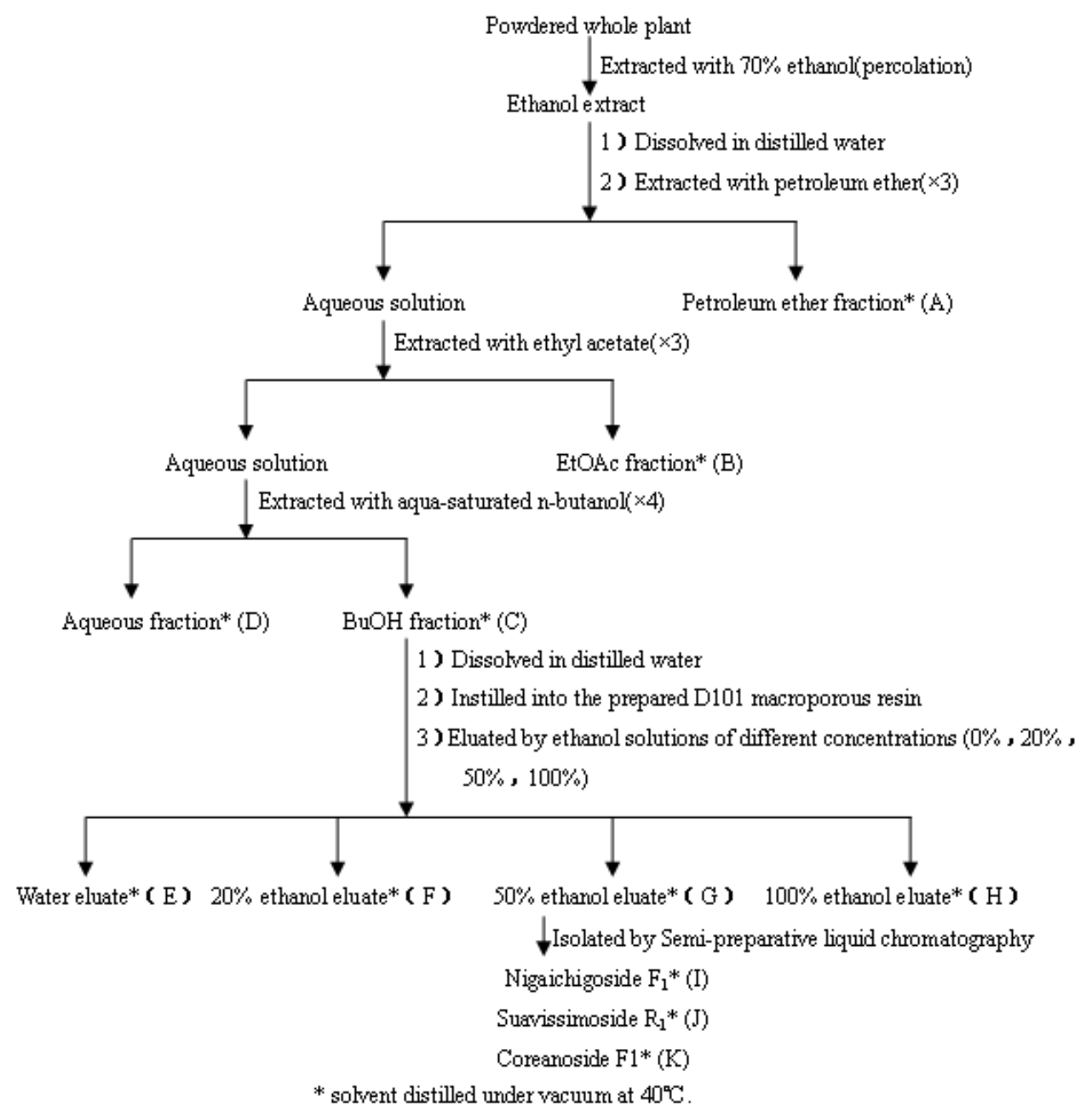


The TLC profile of the $n$-butanol fraction mainly showed the presence of saponins, thus the $n$-butanol fraction was purified and enriched through D101 macroporous resin chromatography. The residue of the $n$-butanol fraction was suspended in distilled water, then the solution was slowly added onto the D101 macroporous resin, at a rate of $2,000 \mathrm{~mL} / \mathrm{h}$, to ensure that the sample was completely adhered to the resin. After that the resin was washed with distilled water until there was no sugar in the elute, and then eluted with ethanol solutions of different concentrations $(20 \%, 50 \%$ and $100 \%)$ successively at $500 \mathrm{~mL} / \mathrm{h}$.

Each desorbed solution was evaporated in vacuo to afford the water eluate (E, $216 \mathrm{~g}$ ), 20\% ethanol eluate (F, $72 \mathrm{~g}), 50 \%$ ethanol eluate $(\mathrm{G}, 88 \mathrm{~g})$ and $100 \%$ ethanol eluate $(\mathrm{H}, 44 \mathrm{~g})$ residues. The $50 \%$ ethanol eluate residue ( $8 \mathrm{~g}$ ) was purified by semi-prep-RP-HPLC with a YMC-Pack ODS-AM column ( $1 \%$ formic acid-methanol, 55:45; flow rate: $2 \mathrm{~mL} / \mathrm{min}$; injection volume: $50 \mu \mathrm{L}$; sample concentration: $100 \mathrm{mg} / \mathrm{mL}$ in methol; UV conditions: $208 \mathrm{~nm}$ ) allowing the isolation of $25 \mathrm{mg}$ of compound I, $16 \mathrm{mg}$ of compound $\mathrm{J}$ and $11 \mathrm{mg}$ of compound K (Figure 1A-D).

$2 \alpha, 3 \beta, 19 \alpha, 23-T e t r a h y d r o x y u r s-12-e n e-28-O-\beta$-D-glucopyranosyl ester (nigaichigoside $F_{1}$, I) (Figure 1C) was obtained as a white powder. ${ }^{1} \mathrm{H}-\mathrm{NMR}\left(500 \mathrm{MHz}, \mathrm{C}_{5} \mathrm{D}_{5} \mathrm{~N}, \delta \mathrm{ppm}\right) 1.61\left(3 \mathrm{H}, \mathrm{s}, \mathrm{CH}_{3}-27\right), 1.40(3 \mathrm{H}$, $\left.\mathrm{s}, \mathrm{CH}_{3}-24\right), 1.28\left(3 \mathrm{H}, \mathrm{s}, \mathrm{CH}_{3}-29\right), 1.22\left(3 \mathrm{H}, \mathrm{s}, \mathrm{CH}_{3}-26\right), 1.05\left(3 \mathrm{H}, \mathrm{s}, \mathrm{CH}_{3}-25\right), 1.02(3 \mathrm{H}, \mathrm{d}, J=6.6 \mathrm{~Hz}$, $\left.\mathrm{CH}_{3}-30\right), 4.53-3.97$ (6H, glucose), 2.88 (1H, s, H-18), 4.25 (1H, m, H-2), 4.75 (1H, d, J=9.0 Hz, H-3), $5.51(1 \mathrm{H}, \mathrm{t}-\mathrm{like}, \mathrm{H}-12), 6.18\left(1 \mathrm{H}, \mathrm{d}, J=7.5 \mathrm{~Hz}, \mathrm{H}-1{ }^{\prime}\right) ;{ }^{13} \mathrm{C}-\mathrm{NMR}\left(125 \mathrm{MHz}, \mathrm{C}_{5} \mathrm{D}_{5} \mathrm{~N}, \delta \mathrm{ppm}\right) 47.2(\mathrm{C}-1)$, 67.9 (C-2), 78.4 (C-3), 43.2 (C-4), 47.3 (C-5), 17.8 (C-6), 32.6 (C-7), 40.4 (C-8), 47.1 (C-9), 36.9 (C-10), 24.1 (C-11), 127.8 (C-12), 138.7 (C-13), 41.5 (C-14), 28.7 (C-15), 26.4 (C-16), 47.9 (C-17), 53.8 (C-18), 72.2 (C-19), 41.5 (C-20), 26.1 (C-21), 37.1 (C-22), 65.9 (C-23), 14.0 (C-24), 17.2 (C-25), 17.1 (C-26), 24.3 (C-27), 176.5 (C-28), 26.6 (C-29), 16.3 (C-30), 95.3 (C-1'), 73.3 (C-2'), 77.9 (C-3'), $70.7\left(\mathrm{C}-4^{\prime}\right), 78.1\left(\mathrm{C}-5^{\prime}\right), 61.8\left(\mathrm{C}-6^{\prime}\right)$.

$2 \alpha, 3 \beta, 19 \alpha$-Trihydroxyurs-12-ene-23-carboxyl-28-O- $\beta$-D-glucopyranosyl ester (suavissimoside $R_{l}, \mathbf{J}$ ) was obtained as a white amorphous powder. ${ }^{1} \mathrm{H}-\mathrm{NMR}\left(500 \mathrm{MHz}, \mathrm{C}_{5} \mathrm{D}_{5} \mathrm{~N}, \delta \mathrm{ppm}\right) 1.73\left(3 \mathrm{H}, \mathrm{s}, \mathrm{CH}_{3}-27\right)$, $1.62\left(3 \mathrm{H}, \mathrm{s}, \mathrm{CH}_{3}-24\right), 1.36\left(3 \mathrm{H}, \mathrm{s}, \mathrm{CH}_{3}-29\right), 1.20\left(3 \mathrm{H}, \mathrm{s}, \mathrm{CH}_{3}-26\right), 1.16\left(3 \mathrm{H}, \mathrm{s}, \mathrm{CH}_{3}-25\right), 1.09(3 \mathrm{H}, \mathrm{d}$, $\left.J=6.6 \mathrm{~Hz}, \mathrm{CH}_{3}-30\right), 2.87(1 \mathrm{H}, \mathrm{s}, \mathrm{H}-18), 4.32(1 \mathrm{H}, \mathrm{m}, \mathrm{H}-2), 4.62(1 \mathrm{H}, \mathrm{d}, J=9.4 \mathrm{~Hz}, \mathrm{H}-3), 5.27(1 \mathrm{H}$, t-like, H-12), $6.27\left(1 \mathrm{H}, \mathrm{d}, J=8.1 \mathrm{~Hz}, \mathrm{H}-1^{\prime}\right) ;{ }^{13} \mathrm{C}-\mathrm{NMR}\left(125 \mathrm{MHz}, \mathrm{C}_{5} \mathrm{D}_{5} \mathrm{~N}, \delta \mathrm{ppm}\right) 48.2(\mathrm{C}-1), 68.3$ (C-2), 80.7 (C-3), 54.2 (C-4), 52.1 (C-5), 21.5 (C-6), 33.3 (C-7), 40.2 (C-8), 47.7 (C-9), 38.3 (C-10), 24.2 (C-11), 127.8 (C-12), 138.8 (C-13), 41.8 (C-14), 28.8 (C-15), 26.1 (C-16), 47.8 (C-17), 53.9 (C-18), 72.3 (C-19), 41.6 (C-20), 26.0 (C-21), 37.2 (C-22), 179.4 (C-23), 13.3 (C-24), 17.1 (C-25), 16.8 (C-26), 24.4 (C-27), 176.3 (C-28), 26.5 (C-29), 16.1 (C-30), 95.5 (C-1'), 73.5 (C-2'), 78.2 (C-3'), $70.9\left(\mathrm{C}-4^{\prime}\right), 78.5\left(\mathrm{C}-5^{\prime}\right), 61.9\left(\mathrm{C}-6^{\prime}\right)$.

Coreanoside $F_{1}(\mathbf{K})$ was obtained as a white powder. ${ }^{1} \mathrm{H}-\mathrm{NMR}\left(500 \mathrm{MHz}, \mathrm{C}_{5} \mathrm{D}_{5} \mathrm{~N}, \delta \mathrm{ppm}\right) 1.05(3 \mathrm{H}, \mathrm{d}$, $\left.J=6.7 \mathrm{~Hz}, \mathrm{CH}_{3}-30^{\prime}\right), 1.09$ (3H, d, $\left.J=6.1 \mathrm{~Hz}, \mathrm{CH}_{3}-30\right), 1.76-1.10$ (9 singlets: $\mathrm{CH}_{3}-29, \mathrm{CH}_{3}-29^{\prime}, \mathrm{CH}_{3}-27$, $\left.\mathrm{CH}_{3}-27^{\prime}, \mathrm{CH}_{3}-26, \mathrm{CH}_{3}-26^{\prime}, \mathrm{CH}_{3}-25, \mathrm{CH}_{3}-25^{\prime}, \mathrm{CH}_{3}-24\right), 2.86$ (1H, s, H-18'), 2.87 (1H, s, H-18), 3.99 $(1 \mathrm{H}, \mathrm{d}, J=10.1 \mathrm{~Hz}, \mathrm{H}-23 \mathrm{~b}), 4.13(1 \mathrm{H}, \mathrm{ddd}, J=4.1,9.4,10.3 \mathrm{~Hz}, \mathrm{H}-2), 4.24(1 \mathrm{H}, \mathrm{d}, J=9.4 \mathrm{~Hz}, \mathrm{H}-3)$, $4.68\left(1 \mathrm{H}, \mathrm{d}, J=10.2 \mathrm{~Hz}, \mathrm{H}-23^{\prime} \mathrm{a}\right), 5.08\left(1 \mathrm{H}, \mathrm{br}, J=10.4 \mathrm{~Hz}, \mathrm{H}-2^{\prime}\right), 5.52$ (1H, t-like, H-12'), 5.53 (1H, t-like, H-12), 6.14 (1H, d, J=7.5 Hz, GlcII-H-1'), 6.15 (1H, d, J=7.3 Hz, GlcI-H-1), 6.51 (1H, br, H-3'). 
For pharmacological studies, fractions A, B, C, D, E, F, G, H, I were dissolved in 1\% aqueous Tween-80. The doses used are expressed as mg of the dried extract per kg body weight.

\subsection{Animals}

Five-week-old male Kunming mice $(20 \pm 2 \mathrm{~g})$ were kept at $25 \pm 1{ }^{\circ} \mathrm{C}$ and $55 \% \pm 10 \%$ relative humidity with $12 \mathrm{~h}$ of light (artificial illumination: 08:00-20:00). Animal study was conducted in accordance with the guidelines for the Ethical Treatment of Laboratory Animals (License No. SYXK (Yu) 2007-0002). All procedures of mouse care and handling were in accordance with accepted standard operating procedures of the Third Military Medical University. Animals study was approved by the Animal Care and Utilization Committee of the Third Military Medical University.

\subsection{Experimental Design}

\subsubsection{Weight-Loaded Swimming Experiment I}

Mice were trained to be accustomed to swimming once (for $10 \mathrm{~min}$ ) in the first week. To reduce the variations in the swimming capacity, mice capable of swimming with $30 \%$ longer or shorter time than the average with similar swimming time and body weights were excluded. Mice were randomly grouped into ten groups of each containing ten animals. Mice in the treatment groups with four RPL fractions (petroleum ether, ethyl acetate, $n$-butanol, and aqueous fractions from the aqueous ethanol extract were administered with two doses, $40 \mathrm{mg} / \mathrm{kg}$ and $20 \mathrm{mg} / \mathrm{kg}$ respectively [4]. The positive experimental groups were treated with red ginseng $(40 \mathrm{mg} / \mathrm{kg})$.

The RPL extracts were administered orally daily for consecutive 30 days. In order to make the animals accustomed to swimming, training (no loaded on the tails) was carried out once every 3 days. Thirty minutes after the last oral administration, the swimming-to-exhaustion test was carried out as previously described [19-21]. Briefly, mice were dropped individually into an acrylic plastic pool $(90 \mathrm{~cm} \times 50 \mathrm{~cm} \times 50 \mathrm{~cm})$ filled with fresh water at $25 \pm 1{ }^{\circ} \mathrm{C}$ at $40 \mathrm{~cm}$ deep so that the mice could not touch the bottom with their tails to support themselves. A lead block ( $4 \%$ of body weight) was attached to the tail root of each animal. The swimming time to exhaustion was used as the index of the forced swimming capacity. The mice were assessed as exhausted when they failed to rise to the surface of water for breath within a $10 \mathrm{~s}$ period.

\subsubsection{Analysis of Blood Biochemical Parameters}

Blood samples were collected without anticoagulant after measurement of the swimming time and cooled for about $3.5 \mathrm{~h}$ at $4{ }^{\circ} \mathrm{C}$. The serum was prepared by centrifugation at a speed of $1,000 \times \mathrm{g}, 4{ }^{\circ} \mathrm{C}$ for $20 \mathrm{~min}$ and the levels of serum urea nitrogen (SUN), serum triglyceride (TG), serum lactate dehydrogenase (LDH) and serum ammonia were quantified using commercial kits supplied by Beckman Coulter, Inc. (Brea, CA, USA). 


\subsubsection{Weight-Loaded Swimming Experiment II}

Based on the similar swimming time and body weights, mice were randomly divided into fourteen groups, including one non-swimming control group, one swimming control group, two positive control groups and ten treatment groups. Each group contained ten mice. The red ginseng treatment positive control groups were administered with $40 \mathrm{mg} / \mathrm{kg}$ and $20 \mathrm{mg} / \mathrm{kg}$ respectively. The treatment groups with the different eluates collected from D101 macroporous resin were gavaged with $20 \mathrm{mg} / \mathrm{kg}$ and $10 \mathrm{mg} / \mathrm{kg}$ eluates respectively [22]. Nigaichigoside $F_{1}$ was applied at 2 or $4 \mathrm{mg} / \mathrm{kg}$. The doses were selected based on the results of preliminary experiments. The measurement of the weight-loaded swimming test and biochemical analysis of serum were similar to experiment I with the exception that extracts of RPL were administered orally daily for a consecutive 15 days and a lead block (3\% of body weight) was loaded on the tail root of the mice. After that, the levels of serum urea nitrogen (SUN), serum triglyceride (TG), serum lactate dehydrogenase (LDH), lactic acid (LA), serum ammonia and hepatic glycogen (HG) were determined by the automatic biochemical analyser with commercial kits. Two inflammatory cytokines, interleukin-6 (IL-6) and tumor necrosis factor (TNF- $\alpha$ ) in the serum were also examined.

\subsection{Statistical Analysis}

All values are expressed as the denotes \pm S.E.M. All statistical analyses were performed using SPSS software (SPSS for Windows 16.0, SPSS Inc., Chicago, IL, USA). Statistical difference was determined by one-way ANOVA, followed by LSD-t test for multigroup comparisons. $p<0.05$ was considered statistically significant.

\section{Conclusions}

To assess the effect of RPL on physical fatigue and identify the effective component(s) in RPL, we carried out bioactivity-guided fractionation and selected a mouse weight-loaded swimming test for examination of the extent of physical fatigue. The study confirms the anti-fatigue effect of RPL and the active components of RPL to be its total saponins. Nigaichigoside $F_{1}$ is at least partly responsible for the pharmacological effect of RPL. The possible mechanisms of alleviation of physical fatigue by RPL may involve delays in the accumulation of SUN and LA, a decrease in the level of TG by increasing fat utilization, increases in the levels of $\mathrm{HG}$ and $\mathrm{LDH}$ to reduce the accumulation of lactic acid, a reduction of the blood ammonia concentration in the muscle, and suppressions of increments of immune activation and inflammatory cytokines. Further studies are needed for isolation of possible novel compounds from RPL with anti-fatigue activity.

\section{Acknowledgments}

This study was supported by the National Natural Science Foundation of China (NSFC, No. 81273608), National Natural Science and Technology Mayor Projects (No. 2011ZXJ09106-06B) and Chongqing Municipal Engineering Technology Research Center Construction Project (No. CSTC2012PT-GC0003). We thank Houhui Huang (Inspection Branch of Southwest Hospital, Chongqing, China) for the technical assistance in analyzing serum biochemical parameters. 


\section{Conflicts of Interest}

The authors declare no conflict of interest.

\section{References}

1. Compilation Group of Chinese Herbal Medicine Compilation. Chinese Herbal Medicine Compilation; People's Medical Publishing House: Beijing, China, 1975; p. 512.

2. Deighton, N.; Brennan, R.; Finn, C.; Davies, H.V. Antioxidant properties of domesticated and wild Rubus species. J. Sci. Food Agric. 2000, 80, 1307-1313.

3. Shyur, L.F.; Tsung, J.H.; Chiu, C.Y.; Lo, C.P. Antioxidant properties of extracts from medicinal plants popularly used inTaiwan. Int. J. Appl. Sci. Eng. 2005, 3, 195-202.

4. Gao, J.; Sun, C.R.; Yang, J.H.; Shi, J.M.; Du, Y.G.; Zhang, Y.Y.; Li, J.H.; Wan, H.T. Evaluation of the hepatoprotective and antioxidant activities of Rubus parvifolius L. J. Zhejiang Univ. Sci. B 2011, 12, 135-142.

5. Wang, J.S.; Li, H.Z.; Qiu, Z.Y.; Xia, Y.P.; Ren, L.Y.; Zhou, C.L. Protective effects of aqueous extract of Rubus parviflolius on middle cerebral artery occlusion and reperfusion injury in rats. Chin. J. New Drugs Clin. Remed. 2006, 12, 920-923.

6. Wang, J.S.; Qiu, Z.Y.; Xia, Y.P.; Li, H.Z.; Ren, L.Y.; Zhang, L. The protective effects of total glycosides Rubus parviflolius on cerebral ischemia in rat. Zhongguo Zhong Yao Za Zhi. 2006, 2, $138-141$.

7. Zheng, Z.X.; Zhang, L.J.; Hang, C.X. Antitumor effects of the total saponins of Rubus parvifolius L. on malignant melanoma. Zhongguo Zhong Yao Za Zhi. 2007, 32, 2055-2058.

8. Akazawa, K.H.; Cui, Y.; Tanaka, M.; Kataoka, Y.; Yoneda, Y.; Watanabe, Y. Mapping of regional brain activation in response to fatigue-load and recovery in rats with c-Fos immunohistochemistry. Neurosci. Res. 2009, 66, 372-379.

9. Chaudhuri, A.; Behan, P.O. Fatigue in neurological disorders. Lancet 2004, 363, 978-988.

10. Huang, L.Z.; Huang, B.K.; Ye, Q.; Qin, L.P. Bioactivity-guided fractionation for anti-fatigue property of Acanthopanax senticosus. J. Ethnopharmacol. 2011, 133, 213-219.

11. Seto, T.; Tanaka, T.; Tanaka, O.; Naruhashi, N. $\beta$-glucosyl esters of $19 \alpha$-hydroxyursolic acid derivatives in leaves of Rubus species. Phytochemistry 1984, 23, 2829-2834.

12. Du, S.H.; Liu, W.Y.; Rao, J.H.; Bai, J. Two isolated saponins from Rubus parvifolius L. on preparative HPLC. Chin. Tradit. Herbal Drugs 2005, 36, 348-350.

13. Nguelefack, T.B.; Mbakam, F.H.K.; Tapondjou, L.A.; Watcho, P.; Nguelefack-Mbuyo, E.P.; Ponou, B.K.; Kamanyi, A.; Park, H.-J. A dimeric triterpenoid glycoside and flavonoid glycosides with free radical-scavenging activity isolated from rubus rigidus var. camerunensis. Arch. Pharm. Res. 2011, 34, 543-550.

14. Wang, J.; Li, S.S.; Fan, Y.Y; Chen, Y.; Liu, D.; Cheng, H.R.; Gao, X.G. Anti-fatigue activity of the water-soluble polysaccharides isolated from panax ginseng C. A. Meyer. J. Ethnopharmacol. 2010, 130, 421-423. 
15. Huang, L.Z.; Huang, B.K.; Liang, J.; Zheng, C.J.; Han, T.; Zhang, Q.Y.; Qin, L.P. Antifatigue activity of the liposoluble fraction from Acanthopanax senticosus. Phytochem. Res. 2011, 25, 940-943.

16. Hamer, M.; Sabia, S.; Batty, G.D.; Shipley, M.J.; Tabàk, A.G.; Singh-Manoux, A.; Kivimaki, M. Physical activity and inflammatory markers over 10 years follow-up in men and women from the whitehall II cohort study. Exerc. Physiol. 2012, 126, 928-933.

17. Cho, H.J.; Kivimaki, M.; Bower, J.E.; Irwin, M.R. Association of C-reactive protein and interleukin-6 with new-onset fatigue in the Whitehall II prospective cohort study. Psychol. Med. 2013, 43, 1773-1783.

18. Sun, R.; Fu, K.; Fu, Y.; Zu, Y.; Wang, Y.; Luo, M.; Li, S.; Luo, H.; Li, Z. Preparative separation and enrichment of four taxoids fromTaxus chinensis needles extracts by macroporous resin column chromatography. J. Sep. Sci. 2009, 32, 1284-1293.

19. Tang, W.; Zhang, Y.; Gao, J.; Ding, X.; Gao, S. The anti-fatigue effect of 20(R)-ginsenoside Rg3 inmice by intranasally administration. Biol. Phar.-Maceutical. Bull. 2008, 31, 2024-2027.

20. Zhang, Y.; Yao, X.; Bao, B. Anti-fatigue activity of a triterpenoid-rich extract from Chinese bamboo shavings (Caulis bamfusae in taeniam). Phytother. Res. 2006, 20, 872-876.

21. Chen, Y.; Kong, L.D.; Xia, X.; Kung, H.F.; Zhang, L. Behavioral and biochemical studies of total furocoumarins from seeds of Psoralea corylifolia in the forced swimming test in mice. J. Ethnopharmacol. 2005, 96, 451-459.

22. Xu, L.R.; Wang, J.S.; Li, H.Z. Effects of Rubus parvifolius L. on neuronal apoptosis and expression of apoptosis-related proteins in a rat model of focal cerebral ischemic-reperfusion injury. Neural Regen. Res. 2008, 3, 742-746.

Sample Availability: Not available.

(C) 2013 by the authors; licensee MDPI, Basel, Switzerland. This article is an open access article distributed under the terms and conditions of the Creative Commons Attribution license (http://creativecommons.org/licenses/by/3.0/). 